\title{
ASTROMETRIC WORK AT THE CAPE OBSERVATORY AND THE KSZ
}

\author{
By R. H. STOY
}

\begin{abstract}
A brief description of the astrometric programmes at the Cape Observatory is followed by a short discussion of the problem of extending the KSZ to the South Pole. It is suggested that much of the work for this project could be done by photography.
\end{abstract}

Three astrometric programmes are being carried out at the Cape Observatory. They are (I) the visual meridian observations, (2) the photographic observations for the determination of star positions and proper motions and (3) the observations for stellar parallax.

Meridian observations have been made continuously at the Cape since 1829 when Fallows began to observe with a 5 -inch Dollond transit and a 4-inch Jones mural circle. These instruments were replaced in $\mathrm{I} 855$ by the 8 -inch Airy transit circle, the twin of the famous Greenwich instrument. The Airy transit circle was used up to I939, but only as a secondary instrument after I905 when the Gill 6-inch reversible transit circle was brought into commission. Thanks to Gill's genius, this transit circle, though now over fifty years old, can still be classified as a modern instrument. The only major alterations that have been made to it are the redesign of the mechanical drive of the impersonal micrometer in I9I I (rebuilt in I95I) and the replacement in I95I of the circle micrometers by photographic recording.

Most of the meridian observations made prior to 1925 have been incorporated in the Albany General Catalogue. Those made since 1925 fall into two groups, absolute observations of general selections of stars and differential observations of stars selected to serve as standards for the reduction of photographic observations. The absolute observations have resulted in three catalogues which have been recently published. The first of these is the Second Cape Catalogue for I925.0 which is based on observations made between 1925 and 1933 and which gives the places of 3627 zodiacal stars and of 8400 stars brighter than 7.5 and south of $-30^{\circ}$. The second is the Third Cape Catalogue for I925.0 which gives the places of 6597 similar stars between $0^{\circ}$ and $-30^{\circ}$ as derived from observations made between 1933 and 1936. The third is the First Cape Catalogue for 1950.0 which gives the places of 5373 PGC and Backlund-Hough stars south of $+35^{\circ}$ from observations made between 1936 and 1944 .

The Second and Third Catalogues for 1925.0 contain over 14,500 of the GC stars south of the equator. The PGC stars were not included as they had been well observed for the First Cape Catalogue for I925.O and were to be reobserved for the First Cape Catalogue for I950.o. The catalogue now being observed, the Second Cape Catalogue for 1950.0, includes all the stars in the GC between $-30^{\circ}$ and $-52^{\circ}$ and between $-80^{\circ}$ and $-90^{\circ}$. It also includes other stars south of $-80^{\circ}$ which have been specially selected for use in the reduction of photographic observations. It is perhaps worth noting that, by the time the present meridian and photographic programmes are completed, most of the GC stars south of the equator will have been observed twice at the Cape since the GC was compiled, the mean epoch of the first set of observations being near 1930, the second near 1950 .

The differential meridian observations that have been made since 1930 are being formed into zone catalogues. These catalogues contain an average of I.25 stars per square degree, evenly spaced and having an average visual magnitude 8.5. The spectral distribution of over 900 such stars between $-72^{\circ}$ and $-80^{\circ}$ is as follows:

$\begin{array}{lllcccc}\text { Sp. Type } & \text { B } & \text { A } & \text { F } & \text { G } & \text { K } & \text { M } \\ \text { Percentage } & 2 & \text { I } 8 & 20 & 22 & 40 & \text { I }\end{array}$

For the more southerly zones, these catalogues also include all the GC stars which were considered as being too bright to be measured satisfactorily on the corresponding photographic plates. The first three catalogues, those for the zones $-30^{\circ}$ to $-35^{\circ},-35^{\circ}$ to $-40^{\circ}$ and $-5^{\circ}$ to $-56^{\circ}$ were observed between I930 and I939 with the Airy transit circle, the fundamental stars being selected from the First Cape Catalogue for I 925.0 . For the zones from $-56^{\circ}$ to $-64^{\circ}$ and from $-64^{\circ}$ to $-80^{\circ}$, the observations were made between 1944 and 1949 with the Gill transit circle using fundamental stars selected from the GC but with places systematically reduced to the $\mathrm{FK}_{3}$ system. For the zone catalogues these observations with the Gill transit circle were reduced differentially, but they were made absolutely together with the normal observations of the sun and planets. Thus the system of the 
fundamental stars can be controlled by the derived night corrections.

The determination of star positions by photographic methods was begun at the Cape in 1885 when the first photographs for the CPD were taken. This project which gave approximate equatorial coordinates for 454,875 stars south of $-19^{\circ}$ was completed in 1900 . Work on the Cape zone of the Carte du Ciel, $-40^{\circ}$ to $-52^{\circ}$, was begun in $\mathrm{I} 892$. The resulting catalogues giving accurate rectangular coordinates for about 500,000 stars were all published by 1927. The associated proper motion work was finished and published before the war. Of special interest are the catalogues which give accurate equatorial coordinates and proper motions for the 4I,000 stars brighter than $9.5 \mathrm{CPD}$.

The current Cape work on the determination of accurate star places by photography was initiated by Sir Harold Spencer Jones in 1930. This programme, which is modelled on Schlesinger's early work at Allegheny and Yale, aims at providing accurate places for an average of 9 to Io stars per square degree in the portion of the sky south of $-30^{\circ}$. The greater number of these stars have visual magnitudes between 7 and ı. Wherever possible, proper motions are being derived by comparison with earlier visual observations. For many of the stars in the more southerly zones no such earlier observation exists. Photographic and photovisual magnitudes are also being obtained while the spectral types for many of the stars which are not in the Henry Draper Catalogue have been supplied through the kind cooperation of the Harvard College Observatory.

Catalogues typed ready for photographic reproduction are now available for

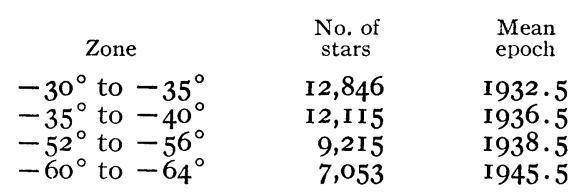

the positions and proper motions being on the $\mathrm{FK}_{3}$ system. Catalogues are now being prepared for the two zones

$$
\begin{array}{lll}
-56^{\circ} \text { to }-60^{\circ} & 7,600 & 1945.5 \\
-64^{\circ} \text { to }-68^{\circ} & 5,000 & \text { I948.0 }
\end{array}
$$

for which all the necessary measures have been made. Through the kindness of the Astronomer Royal, the first of these two zones was measured at Greenwich. All the meridian and photographic observations have been completed for the zones between $-68^{\circ}$ and $-80^{\circ}$, while those for the polar cap south of $-80^{\circ}$ are well in hand and should be finished either this year or early in I954.

The lens that has been used for these observations is $13 \mathrm{~cm}$ in aperture, 2 metres in focal length and gives a scale of $102^{\prime \prime} 3$ per $\mathrm{mm}$. After the plates for the first zone, $-30^{\circ}$ to $-35^{\circ}$, had been taken, the lens was stopped down to $f / 23$ to improve the definition near the edge of the field. For the first two zones, $-30^{\circ}$ to $-35^{\circ}$ and $-35^{\circ}$ to $-40^{\circ}$, a field of $5^{\circ} \times 5^{\circ}$ was measured on each plate, but for all subsequent zones the field measured has been reduced to $4^{\circ} \times 4^{\circ}$ in an attempt to secure greater accuracy. The plates overlap by 50 per cent in right ascension so that each star is measured on two plates.

The first stellar parallax to be determined at the Cape was that of $\alpha$ Centauri which Henderson derived from meridian observations made in I832 and I833. Towards the end of the last century, Sir David Gill and his associates investigated the parallaxes of 22 stars using first a 4-inch and later a 7 -inch heliometer made by Repsold. The precision of the results they obtained witnesses alike to the observers' skill and the instrument-maker's art.

The modern series of Cape stellar parallax observations was commenced by Sir Harold Spencer Jones in 1926. The telescope used, a 24 -inch photographic refractor of $22 \mathrm{ft}$. 6 in. focal length, and the method employed for measuring the plates were similar to those that had been successfully used at Greenwich since I9I3. The programme consisted of stars of large proper motion south of the equator, with stars in the Cape astrographic zone getting a slight preference. Double stars with dynamical parallaxes exceeding $\mathrm{O}$ ".02, stars with large spectroscopic parallaxes or with large parallaxes determined elsewhere were also included. Later, when large gaps were beginning to appear in the original working list, a number of selected $\mathrm{K}$ and $\mathrm{M}$ stars were added in an attempt to find stars of intermediate luminosities.

The observations for the main part of the parallax programme were completed in I95I but a few extra plates are needed to complete the determination of the proper motion in declination. During 1952, the telescope was dismounted for fitting roller bearings to the declination axis and for a new lens cell which should allow a more critical adjustment of the lens. It is not intended 
to resume the parallax observations on the preI950 scale, but it is hoped to continue them for some years to come, confining attention to a comparatively short list of stars of definite astrophysical interest.

Since the start of the programme in 1926 , the parallaxes of I775 stars have been determined; 4000 plates are in hand waiting to be measured for parallax and a few hundred for proper motion. These plates should provide the material for the first determination of the parallaxes of 80 stars and the redetermination of the parallaxes of 90 of the more important stars that were observed earlier in the programme.

So much for the past and present work at the Cape Observatory. What will be the most useful astrometric programmes to undertake when the present ones are completed?

At the moment the most important problem outstanding seems to be the provision of a faint star catalogue such as that discussed at the Rome meeting of the I.A.U. in I952. The essential feature of this catalogue, which is intended to provide a standard system to which all the work that has been and is to be done on faint stars and their proper motions can be reduced, is that it should be homogeneous. It is most desirable that the system of this catalogue should be identical with that used for the bright stars and that it be firmly linked with observations of extragalactic nebulae and other distant objects which can give an absolute check on the proper motion system. It is also desirable that the catalogue should be sufficiently extensive to provide enough standards for the reduction of wide-field photographic plates.

The most detailed proposals put forward at Rome were those of Professor Zverev and his colleagues. Their plan envisages a final homogeneous catalogue (the KSZ) of about 20,000 stars covering the whole sky uniformly and based on a cooperative effort by as many meridian observers as possible. Most of the observations are to be differential from a list of 93I faint stars specially selected as fundamentals. These fundamentals are to be observed by absolute methods and firmly tied into the $\mathrm{FK}_{3}$ system. The KSZ appears to satisfy the desired conditions except that there is some doubt if the suggested number of stars is sufficient for the precise reduction of some wide-field photographic plates.

The I5,355 stars, an average of 12 stars per 25 square degrees, that have been selected for the three-quarters of the sky north of $-30^{\circ}$ have visual magnitudes between 7.5 and 9. I, averaging 8.5, and annual proper motions less than o". 04 and are mostly of spectral types G and K. Doubles and stars with spectral type later than Mo have been avoided. Nearly one-half of the stars are in existing catalogues of precision, such as AGK2A, GC, NZC, etc. To give a strong link with the work that has already been done on the Kapteyn Selected Areas, three or four stars from each of these areas have been included. It is also intended to include a number of comparison stars from each of about 300 regions containing extragalactic nebulae suitable for astrometric purposes.

It will be seen, therefore, that a considerable amount of preparatory work for this project has already been done for the sky north of $-30^{\circ}$. South of this, only the 286 faint fundamental stars have been selected and a provisional list of about 90 extragalactic nebulae and 27 faint Cepheids has been drawn up.

The Cape photographic zone catalogues which are either completely or nearly finished will enable a selection of stars satisfying the adopted criteria to be made between $-30^{\circ}$ and $-68^{\circ}$. South of $-68^{\circ}$, the catalogues will not be completed for five or six years. This portion of the sky contains only I 500 square degrees and would normally be covered by 720 stars. It would be possible to work on twice this number of stars without a serious increase in the size of the programme as a whole. This should ensure that a sufficient number of stars satisfying the proper motion condition are available for retention in the final catalogue. The stars selected as standards for reducing the Cape photographic observations satisfy all the required conditions except that the proper motions of the majority will not be well known before the catalogues are completed, though approximate values can be derived from the observed meridian places. As these stars are over twice as numerous as the stars ultimately required for the $\mathrm{KSZ}$, they might well serve as the provisional selection for this area.

Thus it is now possible to make a selection of stars for the southern part of the KSZ with the exception of those for the nebular regions, which must await an investigation of the individual nebulae. Plates for this purpose can be taken with the two Cape refractors. Many of the nebulae in the provisional list are already included in a survey of selected objects being carried out with the Radcliffe reflector. Assuming 
that the same criteria will be used as for the northern part of the sky, there will be slightly less than 6,000 catalogue stars south of $-30^{\circ}$, even when the suggested extra stars south of $-68^{\circ}$ are included. For the stars south of $-56^{\circ}$, good modern places, post 1945 with probable errors of the order of \pm 0 ". I, will be available. The 3300 stars between $-30^{\circ}$ and $-56^{\circ}$ should, however, be reobserved. This could be done with the Gill transit circle after the programme at present being observed is completed in 1957 . Besides these 3300 stars, the meridian programme should include those of the 93I faint fundamental stars, of the FK 3 stars and of Morgan's N3O stars that are south of $+30^{\circ}$.

It must be emphasized that, while observations with the Gill transit circle will reduce the accidental errors of the individual star places, it will not greatly improve their systematic accuracy since the present system of southern star positions depends very largely on fundamental observations made with this same instrument and must to that extent reproduce those of its individual eccentricities which no amount of repeated observations will completely eliminate. The need for other instruments to make continuous fundamental observations in the southern hemisphere is both obvious and urgent.

The plans for the northern part of the KSZ envisage the cooperation of the meridian instruments of several observatories, so that each star will be observed by more than one instrument. In the case of the fundamental stars, it is estimated that each will be observed nearly fifty times in each coordinate, over half of the observations being absolute ones. In the southern hemisphere there is at present neither the instruments nor the man power available for so thorough or so ambitious a programme of visual observations. It is therefore desirable to consider if there is an alternative method by which similar results could be achieved. An obvious possibility that is worth exploring is the modern wide-field camera.

Following Schlesinger's successful experiments, photographs made on a scale of about $\mathbf{I O O}^{\prime \prime}$ per $\mathrm{mm}$ have come to be accepted as the most efficient method of compiling star catalogues by differential methods. The photographic plate is used to find by interpolation the positions of a large number of stars from those of a relatively few standard stars. From the point of view of the present problem, the important thing to notice is that during this process the errors in the as- sumed places of the standards themselves tend to get smoothed out, since each is compared with the mean system of all the standards on the plate.

What reduction in the accidental errors of the standard stars can we expect to achieve by this method?

The error of a star position that has been found from a photographic plate is a combination of (I) the errors in the assumed places of the standards, (2) the errors of measurement of the star images on the plate and (3) any failure completely to correct for the distortions of the field produced by the lens.

The error introduced by accidental errors in the assumed places of the standards will presumably vary inversely as $(n-p)^{\frac{1}{2}}$ where $n$ is the number of standards used and $p$ is the number of coefficients that have to be determined for the usual plate corrections. The greater the number of standards used, the more accurate the resulting star places, but when $n-p$ is greater than about Io, the rate of increase in accuracy with increasing $n$ ceases to be significant.

For the results from any individual plate, the largest errors are likely to come from the measurement of the images. Unlike the other two errors, these are not entirely systematic so that their effect can be reduced by simple repetition of the plates. The error of measurement varies with the nature of the image so that its average size can be reduced if the measures are confined to that small magnitude range for which the images are an optimum for accurate measurement.

The displacements of the star images from their computed positions which are caused by the distortions introduced by the lens are functions not only of position on the plate but of the size of image and of the colour of the star that produced it. In ordinary zone catalogue work, this correction can be evaluated in two parts from a study of the residuals for the whole zone. The first part is a positional correction and refers to a star of average magnitude and colour, while the second allows for the departure of the individual star from the average. The evidence from several successive zones measured at the Cape is that these corrections stay relatively constant from zone to zone and so presumably from plate to plate.

For the lens used at the Cape, the corrections for distortion are more or less symmetrical around the centre of the field and increase rapidly in size and uncertainty as the edges of the measured 
field are approached. For an investigation in which relatively few stars were involved and for which it would therefore be practicable to multiply the number of plates measured, the uncertainty of the lens distortion correction could be greatly reduced by arranging the observing in zones which overlapped by 50 per cent in declination and in which adjacent plates overlapped by 50 per cent in right ascension. On such a set of plates, each star would be measured four times, once in each quadrant, so that the four corrections which would be applied to the individual measures would tend to cancel out. Incidentally, the star place resulting from the measures of such a set of plates would be referred to a mean of the standards in an area $2 \frac{1}{4}$ times that of an individual plate.

The corrections for lens distortion could be evaluated with greater certainty than in an ordinary zone programme if the stars to be considered were restricted to a relatively small range of colour and magnitude. This could be achieved by suitably selecting the stars. The restriction in colour could also be achieved more adequately and with less restriction on the choice of stars by using a suitable colour filter with a relatively narrow band pass. This latter procedure would be especially useful for observations made at large zenith distances from which the effects of differential atmospheric refraction have to be eliminated. It would only be practicable to use such a filter for routine observations if the aperture of the lens were large enough to photograph stars of the tenth magnitude without exposures of excessive length. This would certainly be the case if the same lens had sufficient aperture to be used without the filter, but with a suitable grating, to transfer the system of these "faint" stars to those stars of the I3th or I4th magnitude of which the positions are required for the observation of the extragalactic nebulae. This suggests that the lens and the filter should be designed for that colour in which the nebulae can be most efficiently observed.

If the 6000 stars proposed for the KSZ south of $-30^{\circ}$ and selected so as to be comparatively uniform in magnitude and colour were to be observed by photographic methods similar to those that have been used in compiling the zone catalogues, it would seem that the accidental errors with which their positions were known could certainly be reduced to the order of \pm 0 ". 07 , and possibly even to \pm 0 ". 05 . This is the equivalent in accuracy to the mean of about $20 \mathrm{ob}$ - servations with a visual meridian instrument. Assuming a plate area of Ioo square degrees, 400 plates would be needed and 24,000 images would have to be measured. If the observations could be made in duplicate using two different lenses, the certainty with which the positions of the stars would be known would be much increased.

Had such a series of observations to be made with the lens that has been used for the Cape zone observations, the number of plates required would be about 2,500 and 50,000 images would have to be measured since the $\mathrm{KSZ}$ stars would have to be supplemented by nearly the same number of stars to get a sufficient number of standards per plate. Even so, the amount of work involved could not be considered as excessive for a project of this importance.

So far, only the smoothing out of the accidental errors in the star places of the faint stars has been considered. The same observations could be used to check the system of the faint stars against that of the bright if the programme plates (or, if need be, special plates) were taken through gratings whose constants were so chosen that the first-order images of suitable bright stars would register on the plate with a density comparable to that of the central images of the faint standard stars. The extra measuring involved in observing these bright stars would be comparatively small.

It might also prove possible to use wide-angle cameras to provide some checks on the systematic accuracy of the faint-star system. One simple check, for example, might be provided by assuming that the scale of the camera remains unaltered while several regions of the sky are photographed on the same plate in quick succession. Another check, by which the right ascension and declination systems could be directly compared, would be possible if the camera could be rotated through $90^{\circ}$ between exposures. This is not impossible, for with the cameras normally used which have a focal length of about two metres and an aperture not exceeding $20 \mathrm{~cm}$, it should not be difficult to mount within roller bearings the whole camera tube with lens and plate holder rigidly attached.

These few tentative suggestions are put forward for discussion, for it does seem that the possibilities of the photographic method should be fully investigated if there is the slightest prospect that it can successfully replace the need for long and relatively expensive meridian programmes. The photographic method has the 